\title{
The Role of Heme and Reactive Oxygen Species in Proliferation and Survival of Trypanosoma cruzi
}

\author{
Marcia Cristina Paes, ${ }^{1,2}$ Daniela Cosentino-Gomes, ${ }^{3,4}$ Cíntia Fernandes de Souza,, 2 \\ Natália Pereira de Almeida Nogueira, ${ }^{1}$ and José Roberto Meyer-Fernandes ${ }^{3,4}$
${ }^{1}$ Laboratório de Interação de Tripanossomatídeos e Vetores, Departamento de Bioquímica, IBRAG, UERJ, 20521-160 Rio de Janeiro, RJ, Brazil
${ }^{2}$ Instituto Nacional de Ciência e Tecnologia-Entomologia Molecular (INCT-EM), 21941-521 Rio de Janeiro, RJ, Brazil
${ }^{3}$ Laboratório de Bioquímica Celular, Instituto de Bioquímica Médica, Centro de Ciências da Saúde, UFRJ, 21941-521 Rio de Janeiro, RJ, Brazil \\ ${ }^{4}$ Instituto Nacional de Ciência e Tecnologia de Biologia Estrutural e Bioimagem, 21941-521 Rio de Janeiro, RJ, Brazil
}

Correspondence should be addressed to Marcia Cristina Paes, mcpaes@uerj.br

Received 8 June 2011; Accepted 15 August 2011

Academic Editor: Xin-zhuan Su

Copyright (C) 2011 Marcia Cristina Paes et al. This is an open access article distributed under the Creative Commons Attribution License, which permits unrestricted use, distribution, and reproduction in any medium, provided the original work is properly cited.

\begin{abstract}
Trypanosoma cruzi, the protozoan responsible for Chagas disease, has a complex life cycle comprehending two distinct hosts and a series of morphological and functional transformations. Hemoglobin degradation inside the insect vector releases high amounts of heme, and this molecule is known to exert a number of physiological functions. Moreover, the absence of its complete biosynthetic pathway in T. cruzi indicates heme as an essential molecule for this trypanosomatid survival. Within the hosts, T. cruzi has to cope with sudden environmental changes especially in the redox status and heme is able to increase the basal production of reactive oxygen species (ROS) which can be also produced as byproducts of the parasite aerobic metabolism. In this regard, ROS sensing is likely to be an important mechanism for the adaptation and interaction of these organisms with their hosts. In this paper we discuss the main features of heme and ROS susceptibility in T. cruzi biology.
\end{abstract}

\section{Trypanosoma cruzi and Its Biological Cycle}

Trypanosoma cruzi comprises a complex group of parasite populations circulating among humans, vectors, reservoirs, and wild and domestic animals [1]. This parasite is the causative agent of Chagas disease or American trypanosomiasis [2] and is transmitted through triatomine vectors, which are blood-sucking insects, when they feed on the vertebrate host.

After an insect feeds on the blood of an infected vertebrate, the development cycle of the parasite begins in the intestinal tract of triatomines. In the anterior midgut, most blood trypomastigotes transform into epimastigotes a few hours after ingestion. Some epimastigotes multiply by longitudinal binary fission, and in the insect rectum, a new differentiation occurs (metacyclogenesis process) in which epimastigotes are transformed into metacyclic trypomastigotes.
These metacyclic trypomastigotes (highly infectious) are shed in feces and reach the bloodstream of a new vertebrate host after this host scratches an insect bite. The organisms penetrate the mucosa where there are many macrophages; after intense multiplication in the host cell in the form of amastigotes, they transform into trypomastigotes again, returning to the vertebrate circulation and completing the cycle [3]. These series of morphological and biochemical transformations in the life cycle may occur in response to external stimuli [4]. Recently, reactive oxygen species (ROS) and heme have been hypothesized to be important signaling molecules. In this way, protozoan parasites, which are specifically located in places where these molecules are constantly released, must evolve special mechanisms to take advantage of them. This paper will focus on the principal features of heme in T. cruzi biology and how different forms of these parasites are susceptible to ROS. 


\section{Vectors of Trypanosoma cruzi}

2.1. Biting Patterns and Importance of the Heme Molecule. Both the Rhodnius prolixus and Triatoma infestans species are very important in disease transmission. Differentiation into each of the five larval stages and the adult stage of these organisms is determined by their food. These bloodsucking insects ingest 6 to 12 times their original weight in blood. Usually, approximately $10 \mathrm{mM}$ of heme bound to hemoglobin is obtained in a single ingestion [5].

Heme is a ubiquitous molecule usually associated with polypeptide chains through interactions between the iron atom and histidine or methionine residues. Heme catalyzes many oxidation processes in biological systems and is very important in cellular functions, as it is involved in oxygen transport (hemoglobin and myoglobin), in cellular respiration (cytochromes), in antioxidant defenses (peroxidases), in drug detoxification enzyme (P450), and cell signalling regulation (nitric oxide synthase) $[6,7]$. Therefore, heme and hemeproteins are involved in basic functions such as oxygen sensitivity, cellular respiration, metabolism, growth, renewal, and cell differentiation, which are all essential for the survival of organisms. Moreover, heme is a toxic molecule due to its ability to generate reactive oxygen species and its amphiphilic features, to associate with lipid membranes, leading to altered membrane permeabilization and cell disruption [8$11]$.

2.2. Heme Uptake by T. cruzi Epimastigotes. The literature reports that there are some organisms that depend on essential hemeproteins but lack a heme biosynthetic pathway in part or in total; trypanosomatids are included in this group $[12,13]$. In fact, biochemical and genomic studies have demonstrated the absence of key enzymes of heme biosynthesis in T. cruzi $[14,15]$. In this regard, it is plausible that these parasites take up heme from the midgut of their vector.

In fact, during the development of T. cruzi epimastigotes in the digestive tract of insects, parasites utilize exogenous growth factors. Using ultrastructural studies, it has been noted that these factors are taken up by endocytosis via the flagellar pocket and the cytostome [16]. The cytostome, present in the anterior region of the cell near the flagellar pocket, is the preferred site of entry of bovine albumin and transferrin, and are reservosomes, the mature form of endosomes [17].

Interestingly, internalization of heme and hemoglobin proceeds through different routes and/or mechanisms. It has been shown through fluorescence microscopy using fluorescent heme analogues that entry is not modified by lowering the temperature, by preincubation with unlabeled hemoglobin or by reduction of ATP production. On the other hand, the transport of heme is compromised by the addition of cyclosporine, an inhibitor of ATP Binding Cassette- (ABC-) type transporters. This is the first evidence that heme uptake involves the activity of a P-glycoprotein (Pgp) homologue, an $\mathrm{ABC}$ transporter [18]. It has also been inferred that other insect trypanosomatids including
Crithidia deanei, Crithidia oncopelti, and Blastocrithidia culicis obtain haem from their bacterial endosymbionts [19].

2.3. The Intracellular Trafficking of Heme in T. cruzi Epimastigotes. Although fluorescent analogue of heme is internalized faster than hemoglobin in T. cruzi, suggesting the existence of two different pathways used to target molecules, the intracellular traffic of heme is the same as other various molecules, including several proteins $[18,20]$. Heme internalization starts at the cytostome, involving vesicles that travel along the cell body (early endosomes), and ends at the reservosomes, which have been described as a site for protein and lipid accumulation [18].

2.4. Hemeas a Signaling Moleculein T. cruzi Proliferation. Malaquias and Oliveira [21] showed that, when exposed to mitogenic factors present in fetal serum, T. cruzi cells are stimulated by phosphoinositide-specific phospholipase $\mathrm{C}$ (PI-PLC), leading to the accumulation of phosphatidylinositol 3-phosphate (IP3) and diacylglycerol (DAG) and increasing their proliferation. Recent studies have shown the importance of second messengers in differentiation of trypanosomatids, including T. brucei-induced cAMP (adenosine $3^{\prime}$ 5' cyclic monophosphate) [22]. Several serine/threonine kinases, including a cyclin-dependent kinases [23] and a cAMP-dependent protein kinase (PKA) [24], phosphatidylinositol 3-kinase [25], a calcium-dependent protein kinase (PKC) [26], and a kinase dependent on calcium/ calmodulin [27], have been identified in T. cruzi epimastigotes through biochemical studies, and in some cases, through molecular studies as well, such as PKA $[28,29]$. The identification of these kinases groups has been corroborated by Parsons et al. [30]. Also in T. cruzi, before the differentiation of epimastigotes into metacyclic trypomastigotes, cAMP levels increase three- to fourfold inducing the differentiation of T. cruzi epimastigotes to metacyclic trypomastigotes [31].

It has been shown that heme, but not hemoglobin or its peptides, stimulates $T$. cruzi proliferation in vitro in a dose-dependent manner. Different strains were tested ( $Y$ and Dm28c), and both increased in the same manner. Further, a wide heme concentration range was employed, and even at higher concentrations, cells proliferated following the heme addition [18]. The authors hypothesized that heme could drive T. cruzi proliferation through a kinase cascade.

Heme-induced growth of epimastigotes is not affected by inhibitors of cGMP-dependent protein kinase (PKG), PKC, PKA, PI3K, or cyclin-dependent kinase. Moreover addition of KN 93 and Myr-AIP (inhibitors of calmodulin kinase) to a culture of these cells reduces the expected growth, indicating the involvement of calmodulin kinase in heme-mediated cell signaling [32]. Furthermore, the authors showed that hemeinduced T. cruzi growth is associated with CaMKII [31], demonstrating a signaling role for the heme molecule in the biological cycle of Trypanosoma cruzi. Recently, heme was shown to modulate a $\left(\mathrm{Na}^{+}+\mathrm{K}^{+}\right)$ATPase, via heme receptor-mediated stimulation of the PI-PLC/PKC signaling pathway in Leishmania amazonensis [33]. On the other hand, in Trypanosoma brucei brucei this biomolecule may 
be involved in nutritional control; it was able to inhibit activity of ectonucleoside triphosphate diphosphohydrolases (E-NTPDases), an enzyme that is involved in the generation of free adenosine outside of the cell, together with ecto- $5^{\prime}$ nucleotidase [34].

2.5. Redox Metabolism of T. cruzi. As a protozoan parasite of vertebrate and invertebrate hosts, $T$. cruzi is susceptible to a number of oxidative killing mechanisms, including reactive oxygen species (ROS). ROS can be produced during the degradation of hemoglobin in the midgut of insect vector as a consequence of the release of high amounts of heme or as a byproduct of T. cruzi aerobic metabolism $[5,35]$. During respiration, molecular oxygen can undergo partial reduction, giving rise to relatively stable species, by accepting one, two, or three electrons, with the formation of superoxide anions $\mathrm{O}_{2}^{--}$, hydrogen peroxide $\left(\mathrm{H}_{2} \mathrm{O}_{2}\right)$, and hydroxyl radicals $\left({ }^{\circ} \mathrm{OH}\right)$, respectively [36]. High rates of $\mathrm{O}_{2}^{--}$can also be produced by the NADPH oxidase complex, which becomes active immediately after phagocytosis by macrophages. Superoxide radicals can also generate the formation of $\mathrm{H}_{2} \mathrm{O}_{2}$ by spontaneous or SOD-catalyzed dismutation [37].

The mitochondria are the main source of ROS generation in most eukaryotic cells. Mitochondrial ROS are recognized as the key element in cell signaling processes and in a variety of degenerative mechanisms [38]. The Trypanosomatidae family is characterized by a single long mitochondrion with a dilated region known as the kinetoplast, in which mitochondrial DNA (kDNA) is observed [39]. Despite these peculiar characteristics, mitochondria of trypanosomatids are able to generate and sustain a membrane potential comparable to mammalian mitochondria [40].

Like other trypanosomatids, T. cruzi has an intricate antioxidant defense system that varies with their life stages and is distinct from its mammal host and insect vector in its complexity. In contrast to their hosts, trypanosomatids lack GSH/glutathione reductase (GR) and thioredoxin/ thioredoxin reductase systems. Their redox metabolism depends on a particular dithiol called trypanothione and its corresponding reductase, trypanothione reductase (TryR). Moreover, T. cruzi lacks catalase and glutathione peroxidase (GPx), two major eukaryotic enzymes employed in the detoxification of peroxides. In spite of the absence of these two enzymes, T. cruzi possesses two peroxiredoxins, an ascorbate-dependent hemoperoxidase, several distinct peroxidases, of which at least two share sequence homology with GPx, and four iron-containing superoxide dismutases (SOD). For a complete review of redox metabolism in $T$. cruzi, see [41]. A controlled balance between extracellular ROS production and the ability of the cell to deal with these oxidants may predict the success of certain life stages in colonization and survival within the host.

\section{Differential Susceptibility of T. cruzi Life Stages to ROS}

T. cruzi responds differently to oxidative stress depending on its life stage. Analysis of 10 strains from T. cruzi demonstrated a significant increase in trypanothione synthetase (TryS) and in cytosolic and mitochondrial tryparedoxin peroxidase isoforms, during differentiation from the noninfective epimastigote to the infective metacyclic trypomastigote form. Moreover, these elevations in antioxidant enzymes were shown to be more pronounced in the virulent strains than in attenuated ones [42]. At the same time, ascorbate peroxidase and TryR remained unchanged during the different life stages of the parasite [42].

These differences in sensitivity correlated with the genetic diversity between the lineages $T$. cruzi. A comparative study between two different strains of T. cruzi, the Tulahuen strain (T. cruzi VI [43], previously classified as T. cruzi I) and the Y strain (T. cruzi II), showed significant differences in the resistance to $\mathrm{H}_{2} \mathrm{O}_{2}$ treatment. Epimastigote forms of Tulahuen strain were shown to be more resistant to $\mathrm{H}_{2} \mathrm{O}_{2}$ than Y strain, presenting higher activity of glucose-6phosphate dehydrogenase (G6PDH), an enzyme implicated in the supply of NADPH, due to proper function of the trypanothione-dependent system and an increase in the cytosolic tryparedoxin peroxidase (TcCPx) content $[35,44]$. Interestingly, Tulahuen strain was shown to be more susceptible to benznidazole, a well known prooxidant trypanocidal drug, than $\mathrm{Y}$ strain $[45,46]$. In agreement with these findings, the T. cruzi I strains Col1.7G2 and Silvio X-10 cl1 displayed more resistance to $\mathrm{H}_{2} \mathrm{O}_{2}$ treatment than the T. cruzi II strains JG and Esmeraldo cl3. Nevertheless, in contrast to the phenotypes observed with the $\mathrm{Y}$ and Tulahuen strains, these differences could not be attributed to differences in the redox potential of the strains analyzed [47]. In this case, the differential sensitivity to oxidative stress was suggested to be due to changes in the activity of MSH2, a central component of the DNA mutation and mismatch repair (MMR) machinery [47]. The MMR has a key function in recognizing and repairing base mismatches and frame shift mismatches that escape DNA polymerase proofreading during DNA replication [48].

With respect to life stage, T. cruzi epimastigotes seem to be more susceptible to the generation of $\mathrm{H}_{2} \mathrm{O}_{2}$ resulting from xanthine oxidase activity in the serum of chagasic patients lacking a complement system. The treatment of epimastigotes with this serum resulted in an inhibition of cell growth in vitro, a decrease in SOD activity, and an increase in membrane lipid peroxidation. Interestingly, the same results were not observed when parasites were treated with serum from healthy individuals [49]. Why epimastigotes have higher susceptibility to human serum than infective forms is unclear, but the exposure of epimastigotes to fresh human serum over a short period of time led to a decrease in cell respiration, loss of mitochondrial membrane potential, increased $\mathrm{O}_{2}^{--}$production, and release of cytochrome $c$, a process characteristic of programmed cell death [50]. Moreover, it seems that the mitochondrion has a fundamental role in epimastigote-dependent complement activation; an accumulation of high amounts of $\mathrm{Ca}^{2+}$ inside the mitochondrial matrix was observed, causing partial dissipation of the inner membrane potential and $\mathrm{O}_{2}^{*-}$ production [51]. In addition to causing an endogenous increase of $\mathrm{O}_{2}^{--}$during contact of epimastigotes with human serum, 
this noninfective stage also triggers $\mathrm{O}_{2}^{--}$formation by macrophages, with high, almost lethal, toxicity to the cell because of the formation of peroxynitrite [52-54]. On the other hand, infective trypomastigotes may be less exposed to peroxynitrite, as these forms may not stimulate the respiratory burst efficiently [54]. Nevertheless, internalization of $T$. cruzi trypomastigotes by macrophages may activate NADPH oxidase, which is involved in $\mathrm{O}_{2}^{--}$production and peroxynitrite formation. These forms also showed a higher sensitivity to peroxynitrite than to $\mathrm{H}_{2} \mathrm{O}_{2}$ [37]. Conversely, Tanaka et al. [55] demonstrated that $\mathrm{H}_{2} \mathrm{O}_{2}$ is the main oxygen metabolite responsible for killing $T$. cruzi inside macrophages, and T. cruzi trypomastigotes were shown to be more resistant to killing by $\mathrm{H}_{2} \mathrm{O}_{2}$ than were the epimastigotes. The $\mathrm{LD}_{50}$ at which epimastigotes were killed was $6.0 \mathrm{nmol} / \mathrm{min} / \mathrm{ml}$ of $\mathrm{H}_{2} \mathrm{O}_{2}$, while the $\mathrm{LD}_{50}$ for trypomastigotes was $8.7 \mathrm{nmol} / \mathrm{min} / \mathrm{ml}$ of $\mathrm{H}_{2} \mathrm{O}_{2}$ in vitro [55]. Exposure of metacyclic trypomastigotes to $70 \mu \mathrm{M} \mathrm{H}_{2} \mathrm{O}_{2}$ for $6 \mathrm{~h}$ caused an increase of 46-fold in G6PDH specific activity, while G6PDH activity from epimastigote forms presented a time-dependent decrease at the same conditions [56]. It seems that trypomastigotes are more resistant to killing by activated primary macrophages or by increased oxygen radicals than epimastigotes [55]. Nevertheless, overexpression of epimastigote TcCPX increased parasite virulence and resistance to macrophage killing [57]. In trypomastigotes, the increased expression of antioxidant enzymes may also be involved to the persistence of these forms in the serum $[41,58,59]$ or inside macrophages during phagocytosis. In agreement with this thought, trypomastigotes with TcCPx overexpressed, caused an increase in parasitemia and tissue inflammation during mouse infections [37]. More severe infections were also observed within metastatic forms of Leishmania; this was suggested to be due to the functional activity of peroxiredoxin [60]. Therefore T. cruzi antioxidant defense could be considered an important virulence factor $[37,57]$.

Although T. cruzi epimastigotes are shown to be more sensitive to ROS, these cells can tolerate various levels of oxidants. Pretreatment of T. cruzi epimastigotes with low $\mathrm{H}_{2} \mathrm{O}_{2}$ concentrations $(15-20 \mu \mathrm{M})$ allowed an increase in cell proliferation of parasites, accompanied by a transient adaptation response to higher $\mathrm{H}_{2} \mathrm{O}_{2}$ concentrations [35]. Moreover, transient oxidative stress can also induce T. cruzi epimastigotes growth by heme stimulation via a mechanism mediated by a CaM Kinase II-like pathway [61]. This adaptation mechanism could be related to increasing expression levels of arginine kinase, an enzyme involved in the interconversion between phosphoarginine, a molecule with high energetic potential like creatine, and ATP. The process was suggested to be independent of redox content, indicating the participation of an unknown stress response mechanism [62].

\section{Redox Metabolism and Drug Resistance}

The drugs currently used against Chagas disease are nifurtimox and benznidazole, two nitro chemotherapeutic agents described to have trypanocidal effects and an ability to generate ROS. ROS generation may occur through the reduction of the nitro group by the action of nitro anion radical or hydronitroxide radical which then may react with molecular oxygen generating $\mathrm{O}_{2}^{\circ-}$. $[63,64]$. Susceptibility of T. cruzi to nifurtimox and benznidazole has been described as correlated with the levels of free and conjugated glutathione $[63,65]$. Treatment of T. cruzi cultures with nifurtimox or benznidazole resulted in a loss of reduced thiol compounds (GSH, trypanothione and glutathionyl spermidine), which was suggested to be probably due to the conjugation of these compounds with reduced metabolites of the nitro drugs rather than an oxidation effect on thiol consumption [65, 66]. Besides, it was also observed that redox-cycling activity of nifurtimox was only acquired at high concentrations doses $(>400 \mu \mathrm{M})$, two orders of magnitude higher than that required for antiproliferative activity [66]. Moreover, nifurtimox has been reported to act as an inhibitor of $T$. cruzi trypanothione reductase, an enzyme responsible for the maintenance of reduced state of the intracellular thiols $[67,68]$. The total amount of free or conjugated glutathione may vary greatly either between different strains of T. cruzi or between the different life stages of a unique strain, with the following differentiation sequence: epimastigote $>$ trypomastigote $>$ amastigote [64]. These differences in thiol contents could explain the diversity in resistance of T. cruzi stages to treatment with these drugs and in its sensibility to ROS. The resistance of $T$. cruzi to trypanocidal drugs can also be associated with higher expression of enzymatic antioxidants. In vitro-induced T. cruzi resistant to benznidazole presented an increase in the expression of the cytosolic and mitochondrial tryparedoxin peroxidase isoforms and in FeSOD isoforms, compared with the correspondent sensitive lineage. [69-71]. Interestingly, these observations were not involved in naturally resistant strains [69-71]. In T. brucei, null mutants for SODB1 (cytosolic, glycosomal) exhibited 3-fold increased susceptibility to nifurtimox than wild-type cells [72]. It seems that nifurtimox and benznidazole divert thiol compounds from their ability to act as free radical scavengers. Therefore the protection against the toxic effects of ROS might rely on enzymatic antioxidant activities. Antioxidant defenses and drug susceptibility of most studied T. cruzi strains are summarized in Table 1.

With respect to the search for alternative drugs for Chagas disease, the activity of natural compounds such as naphthoquinones, natural products of several families of higher plants, has been extensively investigated [73]. Like nifurtimox and benznidazole, the cytotoxicity of naphthoquinones has been implicated in redox cycling and ROS generation [74]. Recently, three derivative compounds of C-allyl lawsone (2-hydroxy-3-allyl-1,4-naphthoquinone) were shown to be effective against intracellular amastigotes, decreasing the percentage of infection in murine macrophages, with low toxicity to host cells. Indeed, it seems that this compound is involved in mitochondrial damage, accompanied by an increase in $\mathrm{H}_{2} \mathrm{O}_{2}$ generation. Epimastigotes were shown to be more resistant than trypomastigotes to treatment with these compounds, despite having a more sensitive mitochondrion and higher accumulation of $\mathrm{H}_{2} \mathrm{O}_{2}$ within the cells [75]. Another type of naphthoquinone, an 
TABle 1: Antioxidant defenses and drug susceptibility of T. cruzi strains.

\begin{tabular}{|c|c|c|c|c|}
\hline Strain & DTU* & Redox state & $\begin{array}{c}\text { Drug } \\
\text { susceptibility }\end{array}$ & Reference \\
\hline Y & II & $\begin{array}{l}\text { Low concentrations of antioxidant } \\
\text { enzymes; lower activity of G6PDH }\end{array}$ & Resistant & {$[42,44]$} \\
\hline Tulahuen & VI & $\begin{array}{l}\text { High concentrations of antioxidant } \\
\text { enzymes; higher activity of G6PDH; } \\
\text { lower Thiol contents }\end{array}$ & Susceptible & {$[42,44,65]$} \\
\hline Colombiana & I & $\begin{array}{l}\text { High concentrations of antioxidant } \\
\text { enzymes; no alteration of TcCPX and } \\
\text { TcMPX and FeSOD isoforms }\end{array}$ & Resistant (natural) & {$[42,69-71]$} \\
\hline Cl Brener & VI & $\begin{array}{l}\text { No alteration of TcCPX and TcMPX } \\
\text { and FeSOD isoforms }\end{array}$ & $\begin{array}{l}\text { Susceptible (in } \\
\text { vitro) }\end{array}$ & {$[69-71]$} \\
\hline
\end{tabular}

* Discrete typing units (DTUs), according to Zingales et al., 2009 [43].

$\alpha$-lapachone derivative, was shown to have a trypanocidal effect. This compound was tested against two strains of $T$. cruzi, the Y strain (T. cruzi II) and the Colombian strain ( $T$. cruzi I), which presented higher resistance to the treatment than Y. In line with these findings, the Y strain is partially resistant and the Colombian strain is highly resistant to the chemotherapeutic agents currently in use. In contrast with the other naphthoquinones, this $\alpha$-lapachone derivative does not have the capacity to generate free radicals, instead, it might function in the inhibition of proteinases [76]. Although sensibility to ROS may be related to the different T. cruzi groups, in vitro analysis of prooxidant drugs susceptibility between T. cruzi I and T. cruzi II strains showed no significant differences $[45,46]$. However, correlation between the susceptibility to benznidazole in distinct genetic groups of T. cruzi has been described [77]. The subject is still controversial, and in this case, geographic distribution and phylogenetic distances of parasites must be considered [45].

\section{Conclusions}

T. cruzi epimastigotes present divergent behavior after exposure to oxidative stress. It seems that these cells can deal with external addition of $\mathrm{H}_{2} \mathrm{O}_{2}$, on the other hand, the generation of ROS from mammalian sites like serum and the immune system represents a challenge to these cells. For example, membrane-bound phosphatases from T. cruzi are more resistant to the addition of sublethal doses of hydrogen peroxide than Trypanosoma rangeli phosphatase [78]. Interestingly, the concentrations of trypanothione vary between 1.52$2.1 \mathrm{mM}$ in epimastigotes, $0.5 \mathrm{mM}$ in trypomastigotes, and $0.12 \mathrm{mM}$ in amastigotes [41]. At the same time, there is an increase in the expression of antioxidant enzymes during the differentiation of $T$. cruzi from a noninfective form to the infective form, trypomastigotes [41, 58]. In contrast, stress-induced oxidant resistance in Leishmania chagasi is not accompanied by an increase in ROS scavengers, but instead is suggested to be associated with heat shock proteins like HSP70 [79, 80]. The mechanism by which T. cruzi escape from the oxidative burst of mammalian macrophages is still unknown; nevertheless, exposure of phosphatidylserine on the surface of trypomastigotes surface induces a deactivating effect on macrophages. This profile is also seen in apoptotic cells as a means to avoid anti-inflammatory responses [67].

There is a growing interest in understanding how ROS could be involved in the signaling process that permits parasites to colonize such different microenvironments. In this way, especially for T. cruzi, the extreme heterogeneity of this population and its susceptibility to oxygen metabolites must be carefully noted. These observations, in addition to their epidemiological significance, could permit the development of more effective drugs for the treatment of Chagas disease.

\section{Acknowledgments}

This work was supported by Fundação de Amparo à Pesquisa do Estado do Rio de Janeiro (FAPERJ) and Conselho Nacional de Desenvolvimento Científico e Tecnológico (CNPq).

\section{References}

[1] E. S. Garcia, N. A. Ratcliffe, M. M. Whitten, M. S. Gonzalez, and P. Azambuja, "Exploring the role of insect host factors in the dynamics of Trypanosoma cruzi-Rhodnius prolixus interactions," Journal of Insect Physiology, vol. 53, no. 1, pp. 11-21, 2007.

[2] C. Chagas, "Nova tripanomíase humana. Estudos sobre a morfologia e o ciclo evolutivo do Schizotrypanum cruzi, n. gen., agente etiológico de nova entidade mórbida do homem," Memórias do Instituto Oswaldo Cruz, vol. 1, pp. 159-218, 1909.

[3] A. Rassi, A. Rassi, and J. A. Marin-Neto, "Chagas disease," The Lancet, vol. 375, no. 9723, pp. 1388-1402, 2010.

[4] D. J. Steenkamp, "Trypanosomal antioxidants and emerging aspects of redox regulation in the trypanosomatids," Antioxidants and Redox Signaling, vol. 4, no. 1, pp. 105-121, 2002.

[5] A. V. Graça-Souza, C. Maya-Monteiro, G. O. Paiva-Silva et al., "Adaptations against heme toxicity in blood-feeding arthropods," Insect Biochemistry and Molecular Biology, vol. 36, no. 4, pp. 322-335, 2006.

[6] P. Ponka, "Cell biology of heme," The American Journal of the Medical Sciences, vol. 318, no. 4, pp. 241-256, 1999.

[7] Y. Zhu and R. B. Silverman, "Revisiting heme mechanisms. A perspective on the mechanisms of nitric oxide synthase (NOS), heme oxygenase (HO), and cytochrome P450s (CYP450s)," Biochemistry, vol. 47, no. 8, pp. 2231-2243, 2008. 
[8] S. W. Ryter and R. M. Tyrrell, "The heme synthesis and degradation pathways: role in oxidant sensitivityHeme oxygenase has both pro- and antioxidant properties," Free Radical Biology and Medicine, vol. 28, no. 2, pp. 289-309, 2000.

[9] L. J. Deterding, D. C. Ramirez, J. R. Dubin, R. P. Mason, and K. B. Tomer, "Identification of free radicals on hemoglobin from its self-peroxidation using mass spectrometry and immunospin trapping: observation of a histidinyl radical," The Journal of Biological Chemistry, vol. 279, no. 12, pp. 11600-11607, 2004.

[10] R. N. Hasan and A. I. Schafer, "Hemin upregulates Egr-1 expression in vascular smooth muscle cells via reactive oxygen species ERK-1/2-Elk-1 and NF- $\kappa \mathrm{B}$," Circulation Research, vol. 102, no. 1, pp. 42-50, 2008.

[11] T. H. Schmitt, W. A. Frezzatti, and S. Schreier, "Hemininduced lipid membrane disorder and increased permeability: a molecular model for the mechanism of cell lysis," Archives of Biochemistry and Biophysics, vol. 307, no. 1, pp. 96-103, 1993.

[12] J. Campos-Salinas, M. Cabello-Donayre, R. García-Hernández et al., "A new ATP-binding cassette protein is involved in intracellular haem trafficking in Leishmania," Molecular Microbiology, vol. 79, no. 6, pp. 1430-1444, 2011.

[13] K. E. J. Tripodi, S. M. Menendez Bravo, and J. A. Cricco, "Role of heme and heme-proteins trypanosomatid essential metabolic pathways," Enzyme Research, vol. 2011, Article ID 873230, 12 pages, 2011.

[14] M. E. Lombardo, L. S. Araujo, and A. Batlle, "5-Aminolevulinic acid synthesis in epimastigotes of Trypanosoma cruzi," International Journal of Biochemistry and Cell Biology, vol. 35, no. 8, pp. 1263-1271, 2003.

[15] N. M. El-Sayed, P. J. Myler, D. C. Bartholomeu et al., "The genome sequence of Trypanosoma cruzi, etiologic agent of chagas disease," Science, vol. 309, no. 5733, pp. 409-435, 2005.

[16] W. De Souza, T. U. De Carvalho, M. Benchimol, and E. Chiari, "Trypanosoma cruzi: ultrastructural, cytochemical and freezefracture studies of protein uptake," Experimental Parasitology, vol. 45, no. 1, pp. 101-115, 1978.

[17] I. Porto-Carreiro, M. Attias, K. Miranda, W. De Souza, and N. Cunha-E-Silva, "Trypanosoma cruzi epimastigote endocytic pathway: cargo enters the cytostome and passes through an early endosomal network before storage in reservosomes," European Journal of Cell Biology, vol. 79, no. 11, pp. 858-869, 2000.

[18] F. A. Lara, C. Sant'Anna, D. Lemos et al., "Heme requirement and intracellular trafficking in Trypanosoma cruzi epimastigotes," Biochemical and Biophysical Research Communications, vol. 355, no. 1, pp. 16-22, 2007.

[19] L. Kořený, J. Lukeš, and M. Oborník, "Evolution of the haem synthetic pathway in kinetoplastid flagellates: an essential pathway that is not essential after all?" International Journal for Parasitology, vol. 40, no. 2, pp. 149-156, 2010.

[20] W. De Souza, "Basic cell biology of Trypanosoma cruzi," Current Pharmaceutical Design, vol. 8, no. 4, pp. 269-285, 2002.

[21] A. T. Malaquias and M. M. Oliveira, "Phospholipid signalling pathways in Trypanosoma cruzi growth control," Acta Tropica, vol. 73, no. 2, pp. 93-108, 1999.

[22] M. Parsons and L. Ruben, "Pathways involved in environmental sensing in trypanosomatids," Parasitology Today, vol. 16, no. 2, pp. 56-62, 2000.

[23] E. B. Gómez, M. I. Santori, S. Laría et al., "Characterization of the Trypanosoma cruzi Cdc2p-related protein kinase 1 and identification of three novel associating cyclins," Molecular and Biochemical Parasitology, vol. 113, no. 1, pp. 97-108, 2001.
[24] C. M. Ochatt, R. M. Ulloa, H. N. Torres, and M. T. Tellez-Inon, "Characterization of the catalytic subunit of Trypanosoma cruzi cyclic AMP-dependent protein kinase," Molecular and Biochemical Parasitology, vol. 57, no. 1, pp. 73-81, 1993.

[25] A. C. Schoijet, K. Miranda, W. Girard-Dias et al., "A Trypanosoma cruzi phosphatidylinositol 3-kinase ( $\mathrm{TcVps} 34)$ is involved in osmoregulation and receptor-mediated endocytosis," The Journal of Biological Chemistry, vol. 283, no. 46, pp. 31541-31550, 2008.

[26] M. L. Gómez, C. M. Ochatt, M. G. Kazanietz, H. N. Torres, and M. T. Téllez-Iñón, "Biochemical and immunological studies of protein kinase C from Trypanosoma cruzi," International Journal for Parasitology, vol. 29, no. 7, pp. 981-989, 1999.

[27] S. B. Ogueta, G. C. Macintosh, and M. T. Téllez-Iñon, "Stagespecific substrate phosphorylation by a Ca2+/calmodulindependent protein kinase in Trypanosoma cruzi," Journal of Eukaryotic Microbiology, vol. 45, no. 4, pp. 392-396, 1998.

[28] H. Huang, C. Werner, L. M. Weiss, M. Wittner, and G. A. Orr, "Molecular cloning and expression of the catalytic subunit of protein kinase A from Trypanosoma cruzi," International Journal for Parasitology, vol. 32, no. 9, pp. 1107-1115, 2002.

[29] H. Huang, L. M. Weiss, F. Nagajyothi et al., "Molecular cloning and characterization of the protein kinase A regulatory subunit of Trypanosoma cruzi," Molecular and Biochemical Parasitology, vol. 149, no. 2, pp. 242-245, 2006.

[30] M. Parsons, E. A. Worthey, P. N. Ward, and J. C. Mottram, "Comparative analysis of the kinomes of three pathogenic trypanosomatids: Leishmania major, Trypanosoma brucei and Trypanosoma cruzi," BMC Genomics, vol. 6, article 127, 2005.

[31] C. Naula and T. Seebeck, "Cyclic AMP signaling in trypanosomatids," Parasitology Today, vol. 16, no. 1, pp. 35-38, 2000.

[32] C. F. Souza, A. B. Carneiro, A. B. Silveira et al., "Heme-induced Trypanosoma cruzi proliferation is mediated by CaM kinase II," Biochemical and Biophysical Research Communications, vol. 390, no. 3, pp. 541-546, 2009.

[33] E. E. Almeida-Amaral, V. C. Cardoso, F. G. Francioli, and J. R. Meyer-Fernandes, "Leishmania amazonensis: heme stimulates $(\mathrm{Na}++\mathrm{K}+)$ ATPase activity via phosphatidylinositol-specific phospholipase C/protein kinase C-like (PI-PLC/PKC) signaling pathways," Experimental Parasitology, vol. 124, no. 4, pp. 436-441, 2010.

[34] M. S. Leite, R. Thomaz, J. H. M. Oliveira, P. L. Oliveira, and J. R. Meyer-Fernandes, "Trypanosoma brucei brucei: effects of ferrous iron and heme on ecto-nucleoside triphosphate diphosphohydrolase activity," Experimental Parasitology, vol. 121, no. 2, pp. 137-143, 2009.

[35] J. K. Finzi, C. W. M. Chiavegatto, K. F. Corat et al., "Trypanosoma cruzi response to the oxidative stress generated by hydrogen peroxide," Molecular and Biochemical Parasitology, vol. 133, no. 1, pp. 37-43, 2004.

[36] J. F. Turrens, "Oxidative stress and antioxidant defenses: a target for the treatment of diseases caused by parasitic protozoa," Molecular Aspects of Medicine, vol. 25, no. 1-2, pp. 211-220, 2004.

[37] M. N. Alvarez, G. Peluffo, L. Piacenza, and R. Radi, "Intraphagosomal peroxynitrite as a macrophage-derived cytotoxin against internalized Trypanosoma cruzi: consequences for oxidative killing and role of microbial peroxiredoxins in infectivity," The Journal of Biological Chemistry, vol. 286, no. 8, pp. 6627-6640, 2011.

[38] A. J. Kowaltowski, N. C. de Souza-Pinto, R. F. Castilho, and A. E. Vercesi, "Mitochondria and reactive oxygen species," Free Radical Biology and Medicine, vol. 47, no. 4, pp. 333-343, 2009. 
[39] W. de Souza, M. Attias, and J. C. F. Rodrigues, "Particularities of mitochondrial structure in parasitic protists (Apicomplexa and Kinetoplastida)," International Journal of Biochemistry and Cell Biology, vol. 41, no. 10, pp. 2069-2080, 2009.

[40] A. E. Vercesi, C. F. Bernardes, M. E. Hoffmann, F. R. Gadelha, and R. Docampo, "Digitonin permeabilization does not affect mitochondrial function and allows the determination of the mitochondrial membrane potential of Trypanosoma cruzi in situ," The Journal of Biological Chemistry, vol. 266, no. 22, pp. 14431-14434, 1991.

[41] F. Irigoín, L. Cibils, M. A. Comini, S. R. Wilkinson, L. Flohé, and R. Radi, "Insights into the redox biology of Trypanosoma cruzi: trypanothione metabolism and oxidant detoxification," Free Radical Biology and Medicine, vol. 45, no. 6, pp. 733-742, 2008.

[42] L. Piacenza, M. P. Zago, G. Peluffo, M. N. Alvarez, M. A. Basombrio, and R. Radi, "Enzymes of the antioxidant network as novel determiners of Trypanosoma cruzi virulence," International Journal for Parasitology, vol. 39, no. 13, pp. 1455-1464, 2009.

[43] B. Zingales, S. G. Andrade, M. R. S. Briones et al., "A new consensus for Trypanosoma cruzi intraspecific nomenclature: second revision meeting recommends TcI to TcVI," Memorias do Instituto Oswaldo Cruz, vol. 104, no. 7, pp. 1051-1054, 2009.

[44] A. A. Mielniczki-Pereira, C. M. Chiavegatto, J. A. López, W. Colli, M. J. M. Alves, and F. R. Gadelha, "Trypanosoma cruzi strains, Tulahuen 2 and Y, besides the difference in resistance to oxidative stress, display differential glucose-6phosphate and 6-phosphogluconate dehydrogenases activities," Acta Tropica, vol. 101, no. 1, pp. 54-60, 2007.

[45] D. Villarreal, C. Barnabé, D. Sereno, and M. Tibayrenc, "Lack of correlation between in vitro susceptibility to Benznidazole and phylogenetic diversity of Trypanosoma cruzi, the agent of Chagas disease," Experimental Parasitology, vol. 108, no. 1-2, pp. 24-31, 2004.

[46] K. P. Luna, I. P. Hernández, C. M. Rueda, M. M. Zorro, S. L. Croft, and P. Escobar, "In vitro susceptibility of Trypanosoma cruzi strains from Santander, Colombia, to hexadecylphosphocholine (miltefosine), nifurtimox and benznidazole," Biomedica, vol. 29, no. 3, pp. 448-455, 2009.

[47] P. C. Campos, V. G. Silva, C. Furtado et al., "Trypanosoma cruzi MSH2: functional analyses on different parasite strains provide evidences for a role on the oxidative stress response," Molecular and Biochemical Parasitology, vol. 176, no. 1, pp. 816, 2010.

[48] C. R. Machado, L. Augusto-Pinto, R. McCulloch, and S. M. R. Teixeira, "DNA metabolism and genetic diversity in Trypanosomes," Mutation Research, vol. 612, no. 1, pp. 40-57, 2006.

[49] S. M. Hernandez, R. A. Kolliker-Frers, M. S. Sanchez et al., "Antiproliferative effect of sera from chagasic patients on Trypanosoma cruzi epimastigotes. Involvement of xanthine oxidase," Acta Tropica, vol. 109, no. 3, pp. 219-225, 2009.

[50] L. Piacenza, F. Irigoín, M. N. Alvarez et al., "Mitochondrial superoxide radicals mediate programmed cell death in Trypanosoma cruzi: cytoprotective action of mitochondrial iron superoxide dismutase overexpression," Biochemical Journal, vol. 403, no. 2, pp. 323-334, 2007.

[51] F. Irigoín, N. M. Inada, M. P. Fernandes et al., "Mitochondrial calcium overload triggers complement-dependent superoxide-mediated programmed cell death in Trypanosoma cruzi," Biochemical Journal, vol. 418, no. 3, pp. 595-604, 2009.
[52] S. R. Wilkinson, D. J. Meyer, and J. M. Kelly, "Biochemical characterization of a trypanosome enzyme with glutathionedependent peroxidase activity," Biochemical Journal, vol. 352, no. 3, pp. 755-761, 2000.

[53] M. Bergeron and M. Olivier, "Trypanosoma cruzi-mediated IFN- $\gamma$-inducible nitric oxide output in macrophages is regulated by iNOS mRNA stability," The Journal of Immunology, vol. 177, no. 9, pp. 6271-6280, 2006.

[54] M. N. Alvarez, L. Piacenza, F. Irigoín, G. Peluffo, and R. Radi, "Macrophage-derived peroxynitrite diffusion and toxicity to Trypanosoma cruzi," Archives of Biochemistry and Biophysics, vol. 432, no. 2, pp. 222-232, 2004.

[55] Y. Tanaka, H. Tanowitz, and B. R. Bloom, "Growth of Trypanosoma cruzi in a cloned macrophage cell line and in a variant defective in oxygen metabolism," Infection and Immunity, vol. 41, no. 3, pp. 1322-1331, 1983.

[56] M. Igoillo-Esteve and J. J. Cazzulo, "The glucose-6-phosphate dehydrogenase from Trypanosoma cruzi: its role in the defense of the parasite against oxidative stress," Molecular and Biochemical Parasitology, vol. 149, no. 2, pp. 170-181, 2006.

[57] L. Piacenza, G. Peluffo, M. N. Alvarez, J. M. Kelly, S. R. Wilkinson, and R. Radi, "Peroxiredoxins play a major role in protecting Trypanosoma cruzi against macrophage- and endogenously-derived peroxynitrite," Biochemical Journal, vol. 410, no. 2, pp. 359-368, 2008.

[58] A. Parodi-Talice, V. Monteiro-Goes, N. Arrambide et al., "Proteomic analysis of metacyclic trypomastigotes undergoing Trypanosoma cruzi metacyclogenesis," Journal of Mass Spectrometry, vol. 42, no. 11, pp. 1422-1432, 2007.

[59] L. Piacenza, M. N. Alvarez, G. Peluffo, and R. Radi, "Fighting the oxidative assault: the Trypanosoma cruzi journey to infection," Current Opinion in Microbiology, vol. 12, no. 4, pp. 415421, 2009.

[60] N. Acestor, S. Masina, A. Ives, J. Walker, N. G. Saravia, and N. Fasel, "Resistance to oxidative stress is associated with metastasis in mucocutaneous leishmaniasis," Journal of Infectious Diseases, vol. 194, no. 8, pp. 1160-1167, 2006.

[61] M. P. Cupello, C. F. de Souza, C. Buchensky et al., "The heme uptake process in Trypanosoma cruzi epimastigotes is inhibited by heme analogues and by inhibitors of ABC transporters," Acta Tropica. In press.

[62] M. R. Miranda, G. E. Canepa, L. A. Bouvier, and C. A. Pereira, "Trypanosoma cruzi: oxidative stress induces arginine kinase expression," Experimental Parasitology, vol. 114, no. 4, pp. 341-344, 2006.

[63] Y. Repetto, E. Opazo, J. D. Maya, M. Agosin, and A. Morello, "Glutathione and trypanothione in several strains of Trypanosoma cruzi: effect of drugs," Comparative Biochemistry and Physiology, vol. 115, no. 2, pp. 281-285, 1996.

[64] J. D. Maya, Y. Repetto, M. Agosín et al., "Effects of Nifurtimox and benznidazole upon glutathione and trypanothione content in epimastigote, trypomastigote and amastigote forms of Trypanosoma cruzi," Molecular and Biochemical Parasitology, vol. 86, no. 1, pp. 101-106, 1997.

[65] C. Moncada, Y. Repetto, J. Aldunate, M. E. Letelier, and A. Morello, "Role of glutathione in the susceptibility of Trypanosoma cruzi to drugs," Comparative Biochemistry and Physiology, vol. 94, no. 1, pp. 87-91, 1989.

[66] M. Boiani, L. Piacenza, P. Hernández et al., "Mode of action of Nifurtimox and $\mathrm{N}$-oxide-containing heterocycles against Trypanosoma cruzi: is oxidative stress involved?" Biochemical Pharmacology, vol. 79, no. 12, pp. 1736-1745, 2010.

[67] M. C. Jockers-Scherubl, R. H. Schirmer, and R. L. KrauthSiegel, "Trypanothione reductase from Trypanosoma cruzi. 
Catalytic properties of the enzyme and inhibition studies with trypanocidal compounds," European Journal of Biochemistry, vol. 180 , no. 2, pp. 267-272, 1989.

[68] G. B. Henderson, P. Ulrich, A. H. Fairlamb et al., "'Subversive' substrates for the enzyme trypanothione disulfide reductase: alternative approach to chemotherapy of Chagas disease," Proceedings of the National Academy of Sciences of the United States of America, vol. 85, no. 15, pp. 5374-5378, 1988.

[69] F. B. Nogueira, J. C. Ruiz, C. Robello, A. J. Romanha, and S. M. F. Murta, "Molecular characterization of cytosolic and mitochondrial tryparedoxin peroxidase in Trypanosoma cruzi populations susceptible and resistant to benznidazole," Parasitology Research, vol. 104, no. 4, pp. 835-844, 2009.

[70] H. M. Andrade, S. M. F. Murta, A. Chapeaurouge, J. Perales, P. Nirdé, and A. J. Romanha, "Proteomic analysis of Trypanosoma cruzi resistance to benznidazole," Journal of Proteome Research, vol. 7, no. 6, pp. 2357-2367, 2008.

[71] S. M. F. Murta, F. B. Nogueira, P. F. dos Santos et al., "Differential gene expression in Trypanosoma cruzi populations susceptible and resistant to benznidazole," Acta Tropica, vol. 107, no. 1, pp. 59-65, 2008.

[72] S. R. Prathalingham, S. R. Wilkinson, D. Horn, and J. M. Kelly, "Deletion of the Trypanosoma brucei superoxide dismutase gene sodb1 increases sensitivity to nifurtimox and benznidazole," Antimicrobial Agents and Chemotherapy, vol. 51, no. 2, pp. 755-758, 2007.

[73] A. V. Pinto and S. L. De Castro, "The trypanocidal activity of naphthoquinones: a review," Molecules, vol. 14, no. 11, pp. 4570-4590, 2009.

[74] M. Dubin, S. H. Fernandez-Villamil, A. O. Stoppani et al., "Cytotoxicity of beta-lapachone, an naphthoquinone with possible therapeutic use," Medicina, vol. 61, no. 3, pp. 343350, 2001.

[75] R. F. S. Menna-Barreto, R. L. S. Goncalves, E. M. Costa et al., "The effects on Trypanosoma cruzi of novel synthetic naphthoquinones are mediated by mitochondrial dysfunction," Free Radical Biology and Medicine, vol. 47, no. 5, pp. 644-653, 2009.

[76] S. C. Bourguignon, H. C. Castro, D. O. Santos et al., "Trypanosoma cruzi: in vitro activity of Epoxy- $\alpha$-Lap, a derivative of $\alpha$-lapachone, on trypomastigote and amastigote forms," Experimental Parasitology, vol. 122, no. 2, pp. 91-96, 2009.

[77] M. J. De Ornelas Toledo, M. T. Bahia, C. M. Carneiro et al., "Chemotherapy with benznidazole and itraconazole for mice infected with different Trypanosoma cruzi clonal genotypes," Antimicrobial Agents and Chemotherapy, vol. 47, no. 1, pp. 223-230, 2003.

[78] D. Cosentino-Gomes, T. Russo-Abrahão, A. L. Fonseca-deSouza, C. R. Ferreira, A. Galina, and J. R. Meyer-Fernandes, "Modulation of Trypanosoma rangeli ecto-phosphatase activity by hydrogen peroxide," Free Radical Biology and Medicine, vol. 47, no. 2, pp. 152-158, 2009.

[79] M. A. Miller, S. E. McGowan, K. R. Gantt et al., "Inducible resistance to oxidant stress in the protozoan Leishmania chagasi," The Journal of Biological Chemistry, vol. 275, no. 43, pp. 33883-33889, 2000.

[80] J. H. Zarley, B. E. Britigan, and M. E. Wilson, "Hydrogen peroxide-mediated toxicity for Leishmania donovani chagasi promastigotes: role of hydroxyl radical and protection by heat shock," The Journal of Clinical Investigation, vol. 88, no. 5, pp. 1511-1521, 1991. 

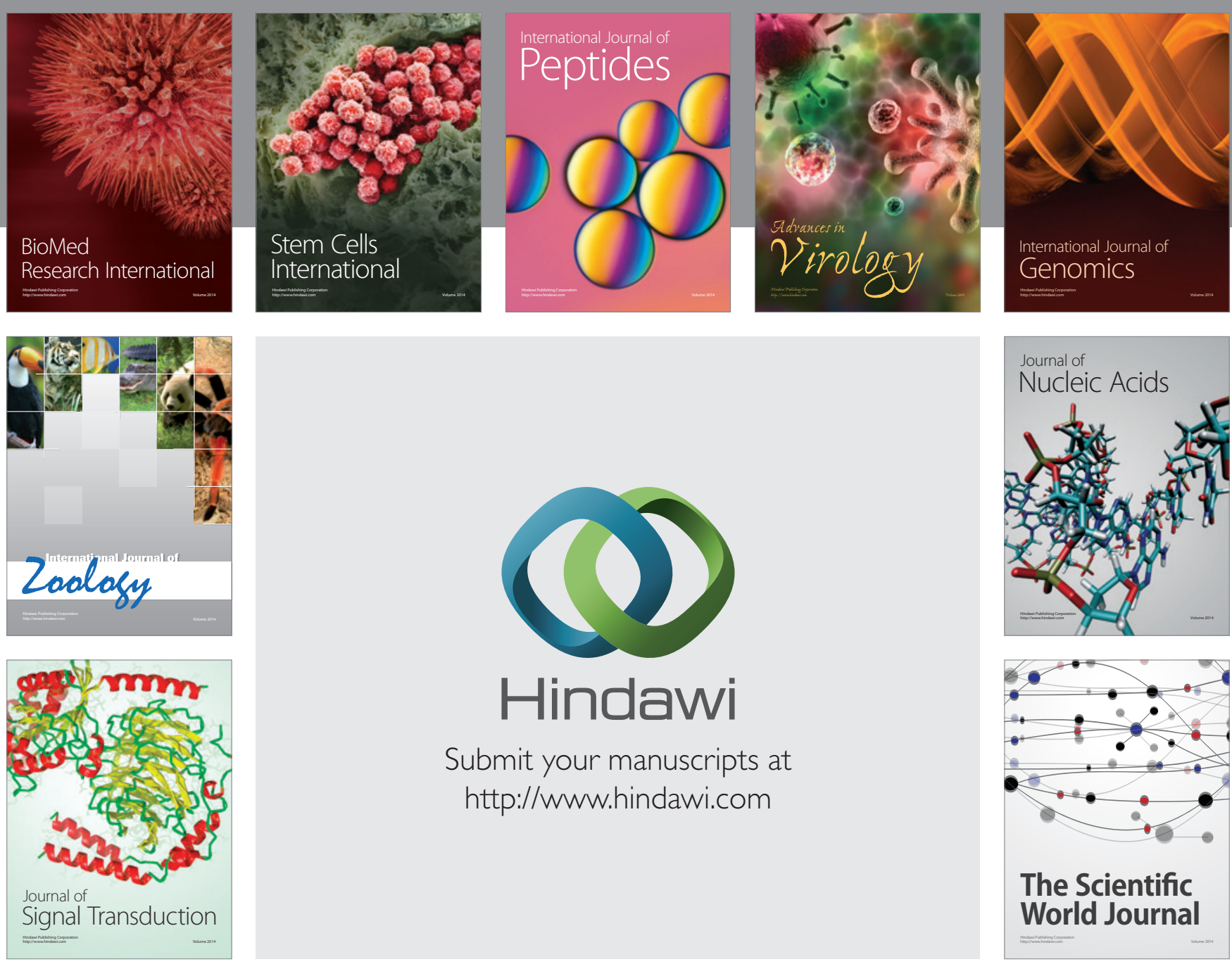

Submit your manuscripts at

http://www.hindawi.com
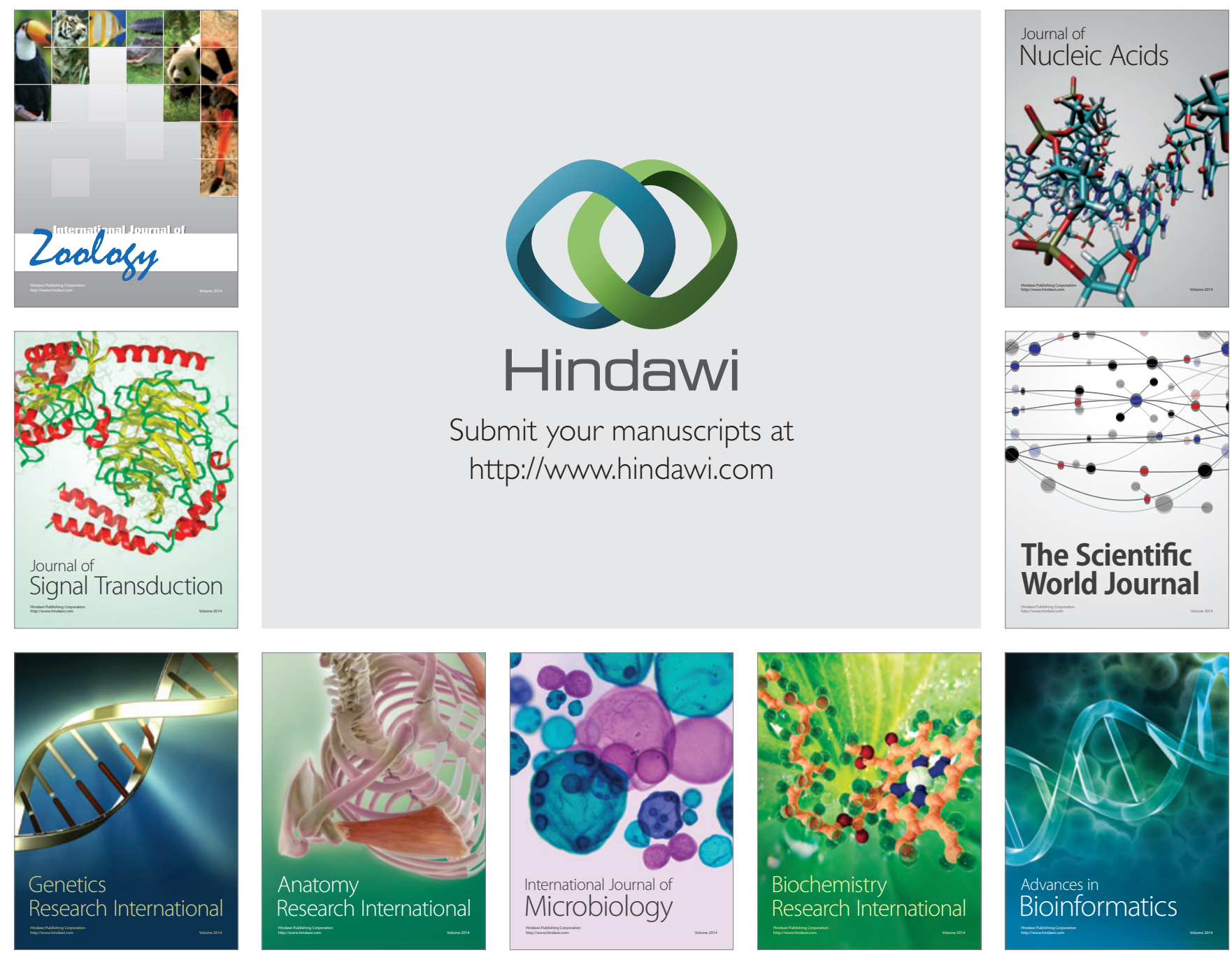

The Scientific World Journal
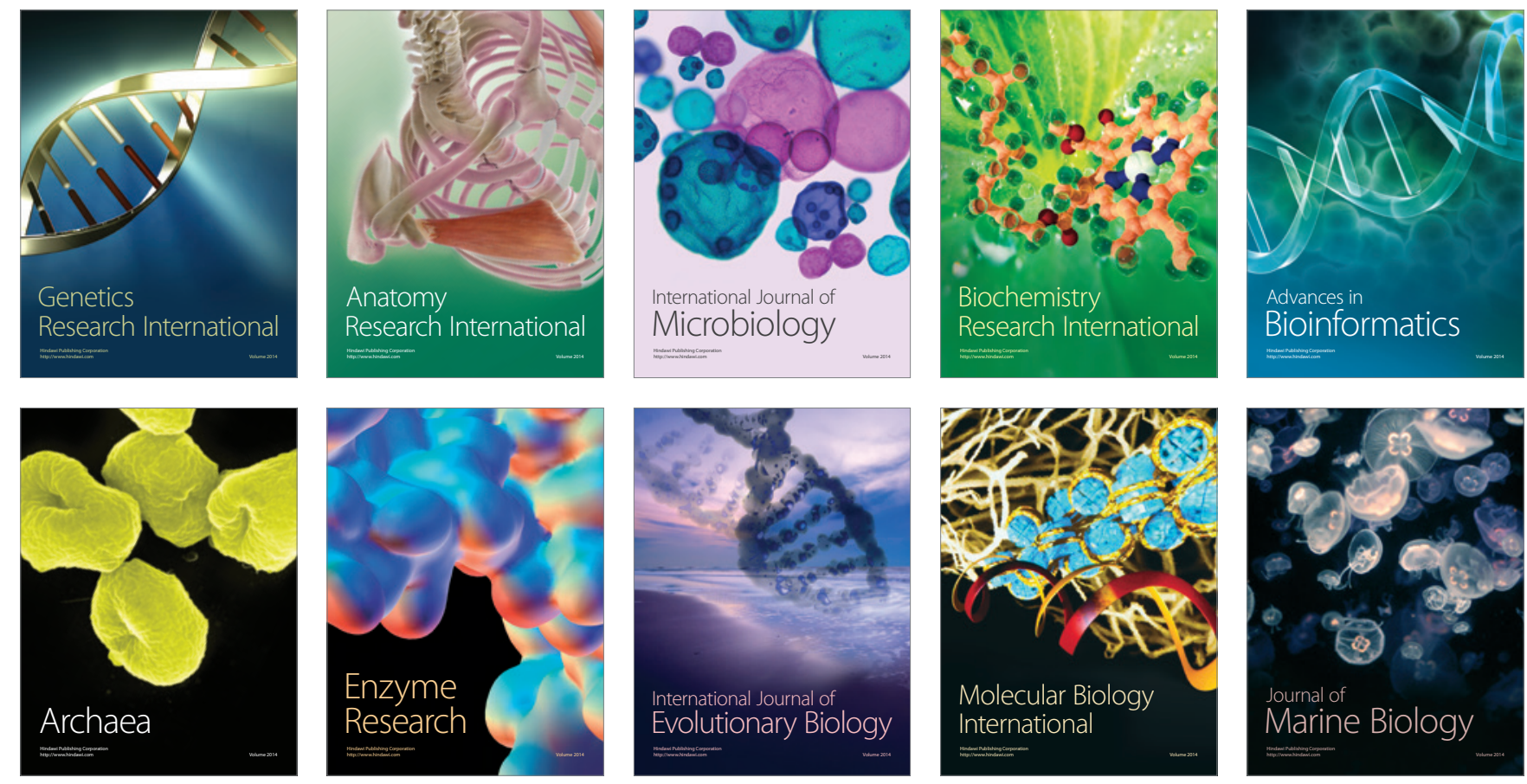